\title{
含 1,3,4-噁二唑啉苯氧乙氧基黄酮衍生物的合成及抗菌活性
}

\author{
段志芳 $*, a, b$ 郡 玲 $a$ \\ $\left({ }^{a}\right.$ 肇庆学院生命科学学院 肇庆 526061) \\ $\left({ }^{b}\right.$ 肇庆学院生物医药工程中心 肇庆 526061)
}

\begin{abstract}
摘要 7-羟基黄酮与过量 1,2 -二溴乙烷反应得到 7-澳乙氧基黄酮, 将其与水杨酫反应得到 7-水杨醛乙氧基黄酮, 然后与 酰胇类化合物反应得到 14 个相应的含酰腙结构的苯氧乙氧基黄酮衍生物，该类化合物与乙酸酐作用分别得到三种类 型的产物, 其中有 8 个为含 1,3,4-堛二唑啉杂环结构的苯氧乙氧基黄酮环合衍生物. 用红外(IR)、核磁共振氢谱( ${ }^{1} \mathrm{H}$ NMR)、质谱(ESI-MS)和高分辨质谱(HR-MS)对化合物的结构进行了确证. 对所合成的化合物进行了初步抗菌活性测定, 结果表明, 在 $0.5 \mathrm{mg} / \mathrm{mL}$ 浓度时, 多数化合物对大肠杆菌、金黄色葡萄球菌、枯草芽孢杆菌具有抑制作用, 而对黑曲霉 抑制作用较弱, 其中 7-(2-对硝基苯甲酰腙基)-苯氧乙氧基黄䣳(4i)对金黄色葡萄球菌和枯草芽狍杆菌作用与对照氯霉 素相近.
\end{abstract}

关键词＼cjkstart酰腙; 1,3,4-噁二唑啉; 黄酮; 合成; 抗菌

\section{Synthesis and Antimicrobial Activity of Flavone Derivatives Containing 1,3,4-Oxadiazoline Structure}

\author{
Duan, Zhifang ${ }^{*, a, b} \quad$ Shao, Ling ${ }^{a}$ \\ ( ${ }^{a}$ College of Life Science, Zhaoqing University, Zhaoqing 526061) \\ ( ${ }^{b}$ Biopharmaceutical Research and Development Center, Zhaoqing University, Zhaoqing 526061)
}

\begin{abstract}
The 7-salicylidene ethoxy flavone was synthesized from salicylaldehyde and 7-bromoethoxy flavones which was obtained by the reaction of 7-hydroxy flavone and 1,2-dibromoethane. Then fourteen flavone derivatives containing acylhydrazone Schiff bases were synthesized by the condensation reaction of this compound with three types of hydrazide compounds, such as benzoylhydrazine, phenoxy acetyl hydrazine and phenylacetyl hydrazine compounds. Finally eight flavone derivatives containing 1,3,4-oxadiazoline structure had been obtained by the cyclodehydration of these hydrazones in acetic anhydride. These new compounds were characterized by IR, ${ }^{1} \mathrm{H}$ NMR, ESI-MS and HRMS. Their antimicrobial activity in vitro was tested. The results showed that at the concentration $0.5 \mathrm{mg} / \mathrm{mL}$ most of the compounds had relatively antibacterial activity, in which 7-(2-p-nitrobenzoyl hydrazone)-phenoxyethoxy flavone (4i) had stronger effects on $S$. aureu and B. subtilis than the others and the activity was similar with that of the control drug chloramphenicol.
\end{abstract}

Keywords acylhydrazone; 1,3,4-oxadiazoline; flavone; synthesis; antimicrobial activity

天然产物黄酮类化合物具有抗氧化、抗肿瘤、抗病 毒等多种药理作用, 对人类肿瘤、衰老、心血管疾病的 防治具有重要意义 ${ }^{[1]}$, 因而此类化合物长久以来倍受人 们关注. 由化学合成和半合成所得到的黄酮衍生物亦具 有广泛的生物活性 ${ }^{[2]}$, 法国里昂公司发现黄酮-8-乙酸具 有抗肿瘤活性 ${ }^{[3]}$, 延等等 ${ }^{[4]}$ 以大豆式元为原料合成了脂 溶性较好的 7-甲氧基-4'-对甲苯磺酰氧基异黄酮和 7-氧
乙酸乙酯-4'-对甲苯磺酰氧基异黄酮, 发现对 1,1-二苯 基-2-三硝基苯朋(DPPH)自由基的清除效果较好; 陈战 国等 ${ }^{[5]}$ 设计合成了 2-甲基苯并咪唑类异黄酮衍生物，具 有较强的清除羟基自由基的能力.

酰腙类化合物含有 $\mathrm{CONHN}=\mathrm{C}$ 活性单元，具有抗 菌、抗肿瘤、抗病毒等生物活性 ${ }^{[6,7]}$, 在农药和医药领域 应用广泛. 其环合产物噁二唑啉化合物是一类重要的杂

*E-mail: zhaoqing606@126.com

Received February 23, 2015; revised April 24, 2015; published online May 8, 2015.

Project supported by the National Natural Science Foundation of China (No. 31201142) and the Research Fund of Science and Technology of Zhaoqing City (No. 2012G25).

国家自然科学基金(No. 31201142)和肇庆市科技基金(No. 2012G25)资助项目. 
环化合物, 具有杀虫、杀菌、抗艾滋病毒等生物活性 ${ }^{[8,9]}$.

本论文为了实现黄酮结构和 1,3,4-惡二唑啉结构生 物活性叠加, 以自制 7-差基黄酮为先导化合物, 与过量 1,2-二溴乙烷反应得到 7-溴乙氧基黄酮, 将其与水杨醛 反应得到 7-水杨醛乙氧基黄酮, 然后通过与三种不同类 型酰肼化合物缩合得到 14 个相应的含酰腙结构的苯氧 乙氧基黄酮衍生物, 此类化合物与乙酸酐作用分别得到 三种类型的产物, 其中 8 个为新型的含 1,3,4-噁二唑啉 杂环的苯氧乙氧基黄酮衍生物, 用红外(IR)、核磁共振 氢谱 $\left({ }^{1} \mathrm{H}\right.$ NMR)、质谱(ESI-MS)及高分辨质谱(HRMS)对 这些化合物的结构进行了确证. 选取革兰氏阴性菌代表 大肠杆菌、革兰氏阳性菌代表金黄色葡萄球菌和枯草杆 菌以及霉菌代表黑曲霉对这些化合物进行了初步抗菌 活性测定. 以期研发具有更强药理作用的黄酮类新药, 为黄酮衍生物的广泛开发和应用提供实验数据. 目标化 合物合成路线如 Scheme 1 所示.

\section{1 结果与讨论}

\section{1 合成}

7-水杨醛乙氧基黄酮与三种类型的酰肼化合物(苯 甲酰肼类、芳乙酰肼类或苯氧乙酰肼类)反应得到 14 个 相应的含酰腙结构的苯氧乙氧基黄酮衍生物, 此类化合 物与乙酸酐作用分别得到三种类型的产物，其中化合物 $4 \mathbf{j} 、 4 k 、 41 、 4 m$ 和 $4 \mathrm{n}$ 与乙酸䣶作用倒入蒸馏水中经后 处理得到的产物为 7-水杨醛乙氧基黄酮, 化合物 $4 \mathrm{~b}$ 与 乙酸䣶作用倒入蒸馏水中经后处理得到的产物为含 1,3,4-噁二唑杂环的苯氧乙氧基黄酮，其余化合物与乙 酸䣶作用经后处理得到的产物为含乙酰化的 1,3,4-噁二 唑啉的苯氧乙氧基黄酮衍生物. 形成原因可能与含苯甲 酰腙结构的苯氧乙氧基黄酮衍生物中 $\mathrm{CH}=\mathrm{NNHCO}$ 受 苯环共轭作用影响比较稳定, 而含乙酰腙结构的苯氧乙 氧基黄酮衍生物中 $\mathrm{CH}=\mathrm{NNHCO}$ 不稳定, 产物易于水 解有关，具体原因有待进一步分析，结合三种反应产物， 推测水解机理为 Scheme 2 .

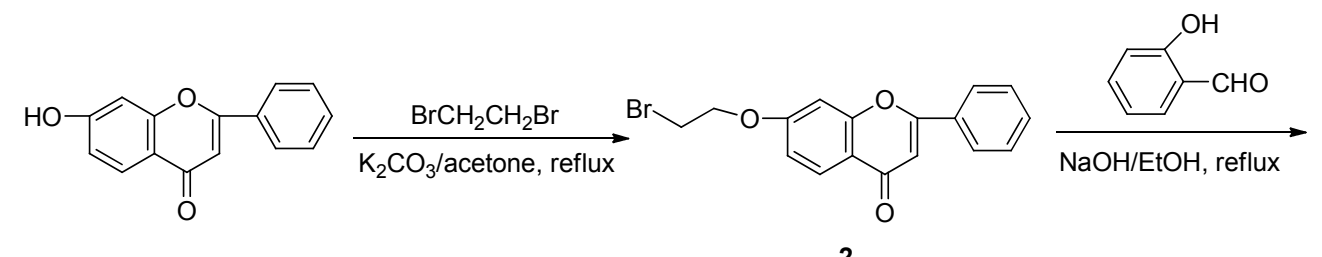

1<smiles>O=Cc1ccccc1OCCOc1ccc2c(=O)cc(-c3ccccc3)oc2c1</smiles>

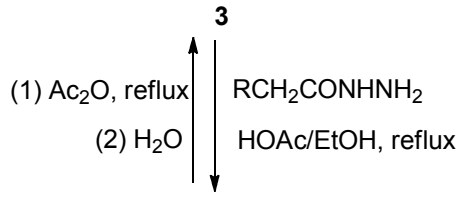<smiles>[R]CC(=O)N/N=C\c1ccccc1OCCOc1ccc2c(=O)cc(-c3ccccc3)oc2c1</smiles>

2

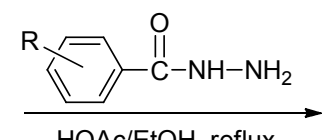

HOAc/EtOH, reflux<smiles>[Z19]C#[W][R]1cccc(C(=O)N/N=C/c2ccccc2OCCOc2ccc3c(=O)cc(-c4ccccc4)oc3c2)c1</smiles>

4a: $\mathrm{R}=\mathrm{H}, \mathbf{4 b}: \mathrm{R}=\mathrm{o}-\mathrm{CH}_{3}, \mathbf{4 c}: \mathrm{R}=m-\mathrm{CH}_{3}$, 4d: $\mathrm{R}=p-\mathrm{CH}_{3}, 4 \mathrm{e}: \mathrm{R}=p-\mathrm{CH}_{3} \mathrm{O}, \mathbf{4 f}: \mathrm{R}=0-\mathrm{HO}$, 4g: $\mathrm{R}=p-\mathrm{HO}, \mathbf{4 h}: \mathrm{R}=p-\mathrm{Cl}, \mathbf{4 i}: \mathrm{R}=p-\mathrm{NO}_{2}$ 1. $\mathrm{Ac}_{2} \mathrm{O}$,reflux 2. $\mathrm{H}_{2} \mathrm{O}$

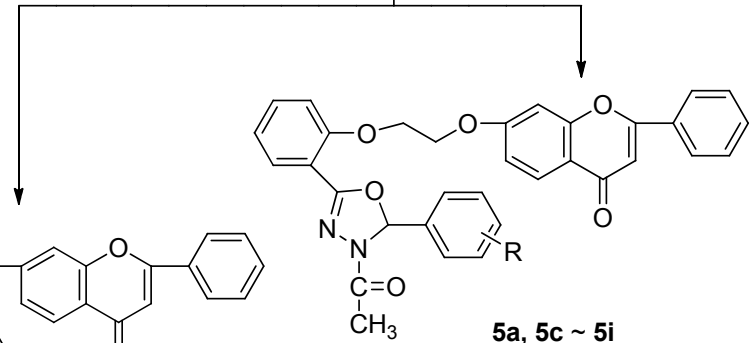

5a: $\mathrm{R}=\mathrm{H}, \mathbf{5 c}: \mathrm{R}=m-\mathrm{CH}_{3}, \mathbf{5 d}: \mathrm{R}=p-\mathrm{CH}_{3}$, 5e: $\mathrm{R}=p-\mathrm{CH}_{3} \mathrm{O}, \mathbf{5 f}: \mathrm{R}=0-\mathrm{CH}_{3} \mathrm{COO}$,

5g: $\mathrm{R}=p-\mathrm{CH}_{3} \mathrm{COO}, \mathbf{5} \mathbf{h}: \mathrm{R}=p-\mathrm{Cl}, \mathbf{5 i}: \mathrm{R}=p-\mathrm{NO}_{2}$

图式 1 含 1,3,4-惡二唑啉苯氧乙氧基黄酮衍生物的合成路线

Scheme 1 Synthetic route of flavone derivatives containing 1,3,4-oxadiazoline structure 


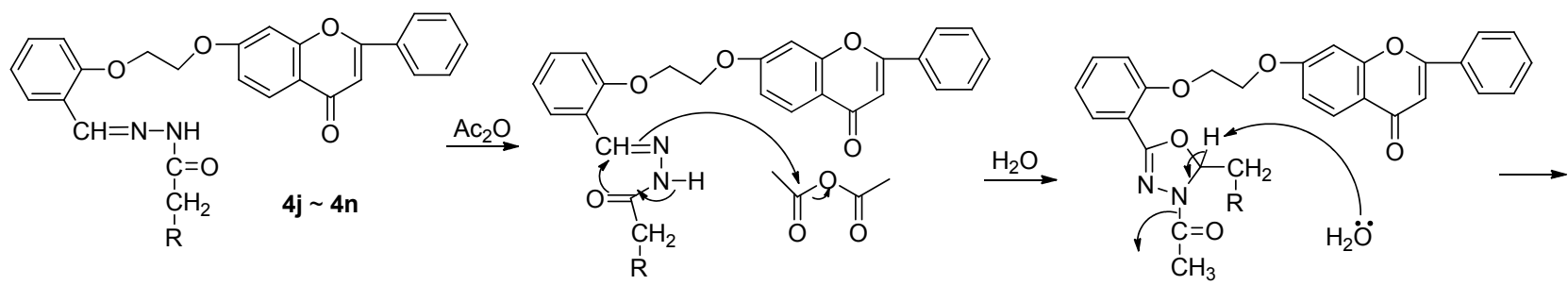<smiles>[R]COc1nnc(-c2ccccc2OCCOc2ccc3c(=O)cc(-c4ccccc4)oc3c2)o1</smiles>

图式 2 水解机理

Scheme 2 Hydrolysis mechanism

\section{2 谱图分析}

通过对所合成化合物的 IR、 ${ }^{1} \mathrm{H} N M R 、{ }^{13} \mathrm{C}$ NMR、 ESI-MS 及 HRMS 图谱数据进行分析, 可以确定目标化 合物结构.

7-(2-取代酰腙基)-苯氧乙氧基黄酮( $4 a \sim 4 n)$ 的 IR 中, $\mathrm{N}-\mathrm{H}$ 的伸缩振动峰在 $3208 \sim 3456 \mathrm{~cm}^{-1}$ 左右; ${ }^{1} \mathrm{H}$ NMR 谱在 $\delta 11.33 \sim 12.12$ 的吸收峰为 $\mathrm{NH}$ 的质子吸收峰, $\delta 8.35 \sim 8.82$ 的吸收峰为 $\mathrm{CH}=\mathrm{N}$ 的质子吸收峰. 在化合 物 $\mathbf{4 j} \sim \mathbf{4 n}$ 的 ${ }^{1} \mathrm{H} N M R$ 谱中 $\mathrm{N}=\mathrm{CH}, \mathrm{NH}$ 和 $\mathrm{CH}_{2}$ 均出现了 成对的峰, 这主要是因为酰腙类化合物分子中的 $\mathrm{CONHN}=\mathrm{C}$ 结构单元同时存在 $E / Z$ 几何异构体和 cis-trans 构象异构体, 参照文献[10]报道, 在 DMSO- $d_{6}$ 作溶剂的条件下 $E$ 式异构体稳定, 谱图中在无偶合情况 下出现的一氢双峰现象是由于 $E$ 式异构体中 cis/trans 转 换造成的, 此两类化合物主要是以 trans 形式存在. 而化 合物 $4 a \sim 4 i$ 则无此现象.

7-\{2-[5-取代苯基-1,3,4-噁二唑(啉)] $\}$-苯氧乙氧基 黄酮 (5a 5i) 的 IR 中 $\mathrm{C}=\mathrm{N}$ 的伸缩振动峰在 $1602 \sim 1610$ $\mathrm{cm}^{-1}$ 左右; ${ }^{1} \mathrm{H}$ NMR 谱在 $\delta 6.78 \sim 8.11$ 之间出现的吸收峰 为芳环上的质子吸收峰, $\delta \quad 6.91 \sim 6.95$ 出现的吸收峰为 黄酮结构上 3 位质子吸收峰, $\delta 4.24 \sim 4.62$ 的吸收峰为 $\mathrm{OCH}_{2} \mathrm{CH}_{2} \mathrm{O}$ 的质子吸收峰; ${ }^{13} \mathrm{C} N \mathrm{NR}$ 谱与化合物结构吻 合; 多数目标化合物的 ESI-MS 中, 有强的准分子离子 峰出现.

以上分析表明目标化合物结构参数与其化学结构 一致.

\section{3 抗菌活性}

采用扩散法(打孔法) 研究了目标化合物在浓度为 $500 \mu \mathrm{g} / \mathrm{mL}$ 时对大肠杆菌 $(E$. coli, $E c)$ 、金黄色葡萄球菌 (S. aureus, $S a)$ 、枯草芽狍杆菌 (B. subtilis, $B s)$ 和黑曲霉 $(A$. niger, $A n$ )的体外抑菌作用, 结果见表 2 (四次测定平均 值).
表 1 化合物浓度为 $0.5 \mathrm{mg} / \mathrm{mL}$ 体外抗菌活性 ${ }^{a}$ Table 1 Antibacterial activity of the compounds at $0.5 \mathrm{mg} / \mathrm{mL}$ in vitro

\begin{tabular}{|c|c|c|c|c|}
\hline Compd. & $E c$ & $S a$ & $B s$ & $A n$ \\
\hline 1 & + & + & + & + \\
\hline 2 & + & + & + & + \\
\hline 3 & ++ & ++ & ++ & ++ \\
\hline $4 a$ & +++ & ++ & + & + \\
\hline $4 b$ & ++ & + & ++ & - \\
\hline $4 c$ & + & + & + & - \\
\hline 4d & + & + & + & - \\
\hline $4 e$ & ++ & + & + & - \\
\hline $4 f$ & + & + & ++ & - \\
\hline $4 \mathrm{~g}$ & ++ & + & + & - \\
\hline $4 h$ & + & ++ & + & + \\
\hline $4 i$ & ++ & +++ & +++ & + \\
\hline $4 j$ & ++ & - & + & ++ \\
\hline $4 k$ & - & + & ++ & ++ \\
\hline 41 & ++ & + & ++ & + \\
\hline $4 m$ & - & - & - & - \\
\hline $4 n$ & - & - & - & - \\
\hline $5 a$ & + & + & + & - \\
\hline $5 \mathbf{b}$ & + & + & ++ & - \\
\hline $5 c$ & + & +++ & + & - \\
\hline $5 d$ & + & + & + & - \\
\hline $5 e$ & + & + & + & - \\
\hline $5 f$ & + & + & ++ & - \\
\hline $5 g$ & + & + & + & - \\
\hline $5 \mathrm{~h}$ & + & + & + & - \\
\hline $5 \mathbf{i}$ & + & + & + & - \\
\hline Chloramphenicol & +++ & +++ & +++ & +++ \\
\hline Blank & - & - & - & - \\
\hline
\end{tabular}

初步抗菌结果显示，多数化合物对黑曲霉抑制作用 较弱, 对大肠杆菌、金黄色葡萄球菌、枯草芽孢杆菌均 具有一定抑制作用，其中 7-(2-苯甲酰腙基)-苯氧乙氧基 黄酮(4a)对大肠杆菌抑制作用较强，7-(2-对硝基苯甲酰 腙基)-苯氧乙氧基黄酮(4i)和 7-[2-(4-乙酰基-5-间甲苯 
基-1,3,4-啞二唑啉)]-苯氧乙氧基黄酮(5c)对金黄色葡萄 球菌抑制作用较强，7-(2-对硝基苯甲酰腙基)-苯氧乙氧 基黄酮(4i)对枯草芽孢杆菌抑制作用较强, 效果与对照 药物氯霉素相近.

\section{2 结论}

本文自制得到三种酰肼类化合物: 苯甲酰肼类、芳 乙酰肼类及苯氧乙酰肼类化合物, 将它们分别与 7-水杨 醛乙氧基黄酮通过缩合反应共得到 14 个含酰腙席夫碱 的黄酮衍生物, 该类化合物与乙酸酐作用得到 1 个含 1,3,4-噁二唑的苯氧乙氧基黄酮衍生物和 8 个含 1,3,4-噁 二唑啉杂环结构的苯氧乙氧基黄酮衍生物. 用红外 (IR)、核磁共振氢谱 $\left({ }^{1} \mathrm{H} N M R\right) 、$ 核磁共振碳谱 $\left({ }^{13} \mathrm{C} \mathrm{NMR}\right)$ 及质谱(ESI-MS)和高分辨质谱(HRMS)对目标化合物的 结构进行了确证. 初步测定了它们对大肠杆菌、金黄色 葡萄球菌、枯草芽狍杆菌和黑曲霉的抑制作用. 生物活 性测试结果表明, 在浓度为 $0.5 \mathrm{mg} / \mathrm{mL}$ 时, 多数化合物 对大肠杆菌、金黄色葡萄球菌、枯草芽孢杆菌具有抑制 作用, 而对黑曲霉抑制作用较弱, 其中化合物 7-(2-对硝 基苯甲酰腙基)-苯氧乙氧基黄酮(4i)对金黄色葡萄球菌 和枯草芽孢杆菌抑制作用均较强. 该结果为进一步研究 含席夫碱结构黄酮类化合物及其环合产物的药理活性 提供了基础数据.

\section{3 实验部分}

\section{1 仪器与试剂}

X-4 型显微熔点测定仪(北京泰克公司, 温度计未校 正); FT-IR 6700 型红外光谱仪(美国 Nicolet 公司, KBr 压 片); Vario EL cube 型元素分析仪(德国 Elementar 公司); Avance AV $500 \mathrm{MHz}$ 超导核磁共振仪(德国 Bruker 公司, 溶剂为 DMSO- $d_{6}$, 内标 TMS); LCQ Deca XP 液质联用 仪(美国 Thermo 公司)、UPLC SQD 质谱仪(美国 Waters)、 LC/MS-IT-TOF 质谱仪(日本岛津公司); ZKJ-1 型循环水 真空百、RE-52AAA 旋转式蒸发器(上海嘉鹏科技有限 公司). 苯甲酰肼类(芳乙酰肼类或苯氧乙酰肼类)化合物 按照参考文献[11]的方法, 由羧酸类化合物制备; 7-着基 黄酮按照参考文献[12]的方法, 由间苯二酚制备; 其余 试剂为国产化学纯或分析纯.

\section{2 实验方法}

\subsubsection{7-溴乙氧基黄酮的合成}

按照参考文献[13]的方法合成. 7-溴乙氧基黄酮(2): 淡黄色颗粒状固体, 产率 73.4\%, m.p. $142 \sim 144{ }^{\circ} \mathrm{C}$; ESI-MS m/z: $345.4\left(\mathrm{M}^{+}, 100\right), 347.3\left(\mathrm{M}^{+}, 98\right.$, 同位素峰).

\subsubsection{7-水杨醛乙氧基黄酮的合成}

在 $250 \mathrm{~mL}$ 圆底烧瓶中加入 $18 \mathrm{mmol}$ 水杨醛、 100 $\mathrm{mL}$ 无水乙醇和 $18 \mathrm{mmol}$ 氢氧化钠, 搅拌溶解后加入 18 $\mathrm{mmol}$ 的 7-溴乙氧基黄酮, 加热回流反应 $16 \mathrm{~h}$ (TLC 跟 踪), 反应完毕后冷却至室温, 抽滤, 用蒸馏水洗涤固 体, 无水乙醇重结晶.

7-水杨醛乙氧基黄酮(3): 淡黄色固体，产率 79.2\%. m.p. $193 \sim 195{ }^{\circ} \mathrm{C} ;{ }^{1} \mathrm{H}$ NMR (DMSO-d $\left.6,500 \mathrm{MHz}\right) \delta$ : 10.37 (s, 1H, CHO), 8.10 (dd, $J=2.20,5.76 \mathrm{~Hz}, 2 \mathrm{H}, \mathrm{ArH}$ ), 7.97 (d, $J=8.84 \mathrm{~Hz}, 1 \mathrm{H}, 5-\mathrm{H}), 7.67 \sim 7.71(\mathrm{~m}, 2 \mathrm{H}, \mathrm{ArH})$, $7.57 \sim 7.62(\mathrm{~m}, 3 \mathrm{H}, \mathrm{ArH}), 7.44(\mathrm{~d}, J=2.32 \mathrm{~Hz}, 1 \mathrm{H}, 8-\mathrm{H})$, $7.34(\mathrm{~d}, J=8.16 \mathrm{~Hz}, 1 \mathrm{H}, \mathrm{ArH}), 7.10 \sim 7.14(\mathrm{~m}, 2 \mathrm{H}, \mathrm{ArH}$, 6-H), 6.97 (s, 1H, 3-H), 4.58 4.62 (m, 4H, $\mathrm{CH}_{2} \mathrm{CH}_{2}$ ); IR (KBr) v: 3068, 3036 (ArH), 2948, $2932\left(\mathrm{CH}_{2}\right), 2867,2767$ $(\mathrm{CH}=\mathrm{O}), 1686(\mathrm{CH}=\mathrm{O}), 1639(\mathrm{C}=\mathrm{O}), 1598,1483,1453$ (Ar), 1373, 1292, 1245, $1178(\mathrm{C}-\mathrm{O}-\mathrm{C}), 1089,1058$, 844, 775, 685, $658 \mathrm{~cm}^{-1}$; ESI-MS m/z: $387.4\left([\mathrm{M}+\mathrm{H}]^{+}\right.$, 100). Anal. calcd for $\mathrm{C}_{24} \mathrm{H}_{18} \mathrm{O}_{5}$ : $\mathrm{C} 74.61, \mathrm{H} \mathrm{4.66}$; found $\mathrm{C}$ 74.78, H 4.52 .

\section{2 .37 -(2-取代酰腙基)一苯氧乙氧基黄酮的合成}

在 $50 \mathrm{~mL}$ 圆底烧瓶中加入 $20 \mathrm{~mL}$ 无水乙醇和 2 mmol 7-水杨醛乙氧基黄酮, 摚拌微热溶解后, 加入 2 $\mathrm{mmol}$ 苯甲酰肼类(芳乙酰肼类或苯氧乙酰肼类)化合物, 滴加几滴冰醋酸, 缓慢升温至回流, TLC 跟踪(展开剂 为 $V_{\text {乙酸乙酯 }}: V_{\text {石油瞵 }}=1: 1$ ), 待原料点 7-水杨醛乙氧基黄 酮消失后停止反应. 静置, 冷却, 抽滤得到固体粗品, 用乙醇或乙醇 $/ N, N$-二甲基甲酰胺重结晶, 得到化合物 $\mathbf{4 a} \sim \mathbf{4 n}$.

7-(2-苯甲酰腙基)-苯氧乙氧基黄酮(4a): 白色固体, 产率 88.8\% m.p. $206 \sim 208^{\circ} \mathrm{C} ;{ }^{1} \mathrm{H}$ NMR (DMSO- $d_{6}, 500$ $\mathrm{MHz}) \delta: 11.91(\mathrm{~s}, 1 \mathrm{H}, \mathrm{NH}), 8.81(\mathrm{~s}, 1 \mathrm{H}, \mathrm{CH}=\mathrm{N}), 8.10(\mathrm{~d}$, $J=7.44 \mathrm{~Hz}, 2 \mathrm{H}, \mathrm{ArH}), 7.97$ (d, $J=8.88 \mathrm{~Hz}, 1 \mathrm{H}, \mathrm{ArH})$, $7.87 \sim 7.93(\mathrm{~m}, 3 \mathrm{H}, \mathrm{ArH}), 7.54 \sim 7.61(\mathrm{~m}, 4 \mathrm{H}, \mathrm{ArH})$, $7.43 \sim 7.50(\mathrm{~m}, 4 \mathrm{H}, \mathrm{ArH}), 7.22(\mathrm{~d}, J=8.36 \mathrm{~Hz}, 1 \mathrm{H}, \mathrm{ArH})$, $7.15 \sim 7.18(\mathrm{dd}, J=1.88,7.04 \mathrm{~Hz}, 1 \mathrm{H}, \mathrm{ArH}), 7.08$ (t, $J=$ $7.64 \mathrm{~Hz}, 1 \mathrm{H}, \mathrm{ArH}), 6.99$ (s, 1H, 3-H), $4.53 \sim 4.56$ (m, 4H, $\mathrm{CH}_{2} \mathrm{CH}_{2}$ ); IR (KBr) v: 3456, 3215, 3067, 2947, 2875, $1645,1632,1603,1547,1491,1441,1369,1292,1242$, 1165, 1093, 845, 760, $683 \mathrm{~cm}^{-1}$; ESI-MS m/z: 105.0 (20), $505.0\left([\mathrm{M}+\mathrm{H}]^{+}, 100\right)$; HRMS calcd for $\mathrm{C}_{31} \mathrm{H}_{25} \mathrm{~N}_{2} \mathrm{O}_{5}[\mathrm{M}+$ $\mathrm{H}]^{+}$505.1758, found 505.1767.

7-(2-邻甲苯甲酰腙基)-苯氧乙氧基)黄酮(4b): 白色 固体，产率 $81.1 \%$. m.p. $196 \sim 198{ }^{\circ} \mathrm{C} ;{ }^{1} \mathrm{H} \mathrm{NMR}$ $\left(\mathrm{DMSO}-d_{6}, 500 \mathrm{MHz}\right) \delta: 11.72(\mathrm{~s}, 1 \mathrm{H}, \mathrm{NH}), 8.68(\mathrm{~s}, 1 \mathrm{H}$, $\mathrm{CH}=\mathrm{N}), 8.08(\mathrm{~d}, J=7.0 \mathrm{~Hz}, 2 \mathrm{H}, \mathrm{ArH}), 7.96(\mathrm{~d}, J=9.0$ 
$\mathrm{Hz}, 1 \mathrm{H}, \mathrm{ArH}), 7.90 \sim 7.92(\mathrm{~m}, 1 \mathrm{H}, \mathrm{ArH}), 7.57 \sim 7.61(\mathrm{~m}$, $3 \mathrm{H}, \mathrm{ArH}), 7.34 \sim 7.45(\mathrm{~m}, 4 \mathrm{H}, \mathrm{ArH}), 7.20 \sim 7.27(\mathrm{~m}, 3 \mathrm{H}$, ArH), $7.12 \sim 7.16(\mathrm{~m}, 1 \mathrm{H}, \mathrm{ArH}), 7.07(\mathrm{t}, J=7.5 \mathrm{~Hz}, 1 \mathrm{H}$, $\operatorname{ArH}), 6.96(\mathrm{~s}, 1 \mathrm{H}, 3-\mathrm{H}), 4.49 \sim 4.53\left(\mathrm{~m}, 4 \mathrm{H}, \mathrm{CH}_{2} \mathrm{CH}_{2}\right)$, 2.35 (s, 3H, $\mathrm{CH}_{3}$ ); IR (KBr) v: 3225, 3054, 2944, 1655, 1636, 1609, $1548 \mathrm{~cm}^{-1}$; ESI-MS m/z: 157.27 (100), 217.20 (41), 236.96 (36), 261.32 (47), $519.28\left([\mathrm{M}+\mathrm{H}]^{+}, 32\right)$, $541.40\left([\mathrm{M}+\mathrm{Na}]^{+}, 32\right)$.

7-(2-间甲苯甲酰腙基)-苯氧乙氧基)黄酮(4c)：白色 固体, 产率 $74.9 \%$. m.p. $180 \sim 182{ }^{\circ} \mathrm{C} ;{ }^{1} \mathrm{H}$ NMR $\left(\right.$ DMSO- $\left.d_{6}, 500 \mathrm{MHz}\right) \delta: 11.82(\mathrm{~s}, 1 \mathrm{H}, \mathrm{NH}), 8.81(\mathrm{~s}, 1 \mathrm{H}$, $\mathrm{CH}=\mathrm{N}), 8.07 \sim 8.09(\mathrm{~m}, 2 \mathrm{H}, \operatorname{ArH}), 7.97(\mathrm{~d}, J=9.0 \mathrm{~Hz}$, 1H, ArH), $7.92(\mathrm{~d}, J=7.5 \mathrm{~Hz}, 1 \mathrm{H}, \mathrm{ArH}), 7.67 \sim 7.69(\mathrm{~m}$, $2 \mathrm{H}, \mathrm{ArH}), 7.56 \sim 7.61(\mathrm{~m}, 3 \mathrm{H}, \mathrm{ArH}), 7.42 \sim 7.46(\mathrm{~m}, 2 \mathrm{H}$, ArH), $7.35 \sim 7.36(\mathrm{~m}, 2 \mathrm{H}, \mathrm{ArH}), 7.22(\mathrm{~d}, J=8.5 \mathrm{~Hz}, 1 \mathrm{H}$, ArH), $7.14 \sim 7.16(\mathrm{~m}, 1 \mathrm{H}, \mathrm{ArH}), 7.06 \sim 7.09(\mathrm{t}, J=7.25$ $\mathrm{Hz}, 1 \mathrm{H}, \operatorname{ArH}), 6.96(\mathrm{~s}, 1 \mathrm{H}, 3-\mathrm{H}), 4.52 \sim 4.56(\mathrm{~m}, 4 \mathrm{H}$, $\mathrm{CH}_{2} \mathrm{CH}_{2}$ ), 2.35 (s, 3H, $\mathrm{CH}_{3}$ ); ESI-MS $m / z$ : 157.14 (100), 179.14 (87), 261.19 (49), $519.47\left([\mathrm{M}+\mathrm{H}]^{+}, 51\right), 541.34$ $\left([\mathrm{M}+\mathrm{Na}]^{+}, 34\right)$.

7-(2-对甲苯甲酰腙基)-苯氧乙氧基黄酮(4d): 白色 固体, 产率 85.3\%. m.p. $227 \sim 229{ }^{\circ} \mathrm{C} ;{ }^{1} \mathrm{H}$ NMR (DMSO- $\left.d_{6}, 500 \mathrm{MHz}\right) \delta: 11.79(\mathrm{~s}, 1 \mathrm{H}, \mathrm{NH}), 8.81(\mathrm{~s}, 1 \mathrm{H}$, $\mathrm{CH}=\mathrm{N}), 8.08 \sim 8.10(\mathrm{dd}, J=1.5,6.5 \mathrm{~Hz}, 2 \mathrm{H}, \mathrm{ArH}), 7.97$ (d, $J=9.0 \mathrm{~Hz}, 1 \mathrm{H}, \mathrm{ArH}), 7.91(\mathrm{~d}, J=7.5 \mathrm{~Hz}, 1 \mathrm{H}, \mathrm{ArH})$, 7.79 (d, $J=8.0 \mathrm{~Hz}, 2 \mathrm{H}, \mathrm{ArH}), 7.56 \sim 7.61(\mathrm{~m}, 3 \mathrm{H}, \mathrm{ArH})$, $7.42 \sim 7.44(\mathrm{~m}, 2 \mathrm{H}, \operatorname{ArH}), 7.21 \sim 7.28(\mathrm{~m}, 2 \mathrm{H}, \operatorname{ArH})$, $7.14 \sim 7.16$ (dd, $J=2.5,6.5 \mathrm{~Hz}, 2 \mathrm{H}, \mathrm{ArH}), 7.08$ (d, $J=7.5$ $\mathrm{Hz}, 1 \mathrm{H}, \operatorname{ArH}), 6.96(\mathrm{~s}, 1 \mathrm{H}, 3-\mathrm{H}), 4.52 \sim 4.56(\mathrm{~m}, 4 \mathrm{H}$, $\mathrm{CH}_{2} \mathrm{CH}_{2}$ ), 2.35 (s, 3H, $\mathrm{CH}_{3}$ ); ESI-MS $m / z$ : 157.01 (96), 179.07 (92), 217.14 (41), 261.19 (48), $519.34\left([\mathrm{M}+\mathrm{H}]^{+}\right.$, 100), $541.27\left([\mathrm{M}+\mathrm{Na}]^{+}, 84\right)$.

7-(2-对甲氧苯甲酰腙基)-苯氧乙氧基黄酮(4e)：白 色固体, 产率 $89.1 \%$. m.p. $214 \sim 216{ }^{\circ} \mathrm{C} ;{ }^{1} \mathrm{H}$ NMR (DMSO-d $\left.d_{6}, 500 \mathrm{MHz}\right) \delta: 11.73$ (s, 1H, NH), 8.79 (s, 1H, $\mathrm{CH}=\mathrm{N}), 8.08 \sim 8.10(\mathrm{dd}, J=1.5,6.5 \mathrm{~Hz}, 2 \mathrm{H}, \mathrm{ArH}), 7.97$ (d, $J=8.5 \mathrm{~Hz}, 1 \mathrm{H}, \mathrm{ArH}), 7.87(\mathrm{~d}, J=9.0 \mathrm{~Hz}, 1 \mathrm{H}, \mathrm{ArH})$, $7.56 \sim 7.62(\mathrm{~m}, 2 \mathrm{H}, \mathrm{ArH}), 7.42 \sim 7.45(\mathrm{~m}, 3 \mathrm{H}, \mathrm{ArH}), 7.21$ (d, $J=8.0 \mathrm{~Hz}, 2 \mathrm{H}, \mathrm{ArH}), 7.14 \sim 7.17$ (dd, $J=2.0,6.5 \mathrm{~Hz}$, $2 \mathrm{H}, \mathrm{ArH}), 7.06(\mathrm{t}, J=7.5 \mathrm{~Hz}, 1 \mathrm{H}, \mathrm{ArH}), 7.00 \sim 7.02(\mathrm{~m}$, $2 \mathrm{H}, \mathrm{ArH}), 6.96(\mathrm{~s}, 1 \mathrm{H}, 3-\mathrm{H}), 4.52 \sim 4.56\left(\mathrm{~m}, 4 \mathrm{H}, \mathrm{CH}_{2} \mathrm{CH}_{2}\right)$, 3.81 (s, 3H, $\mathrm{CH}_{3}$ ); ESI-MS m/z: 157.14 (100), 179.26 (88), $261.32(44), 535.34\left([\mathrm{M}+\mathrm{H}]^{+}, 44\right), 557.34\left([\mathrm{M}+\mathrm{Na}]^{+}\right.$, 36).

7-(2-邻着基苯甲酰腙基)-苯氧乙氧基黄酮(4f): 黄
色固体, 产率 93.3\%. m.p. 258 260 ${ }^{\circ} \mathrm{C} ;{ }^{1} \mathrm{H}$ NMR (DMSO- $\left.d_{6}, 500 \mathrm{MHz}\right) \delta$ : $11.92(\mathrm{~s}, 1 \mathrm{H}, \mathrm{NH}), 11.91(\mathrm{~s}, 1 \mathrm{H}$, $\mathrm{OH}), 8.82(\mathrm{~s}, 1 \mathrm{H}, \mathrm{CH}=\mathrm{N}), 8.07 \sim 8.09(\mathrm{~m}, 2 \mathrm{H}, \mathrm{ArH}), 7.97$ $(\mathrm{d}, J=9.0 \mathrm{~Hz}, 1 \mathrm{H}, \mathrm{ArH}), 7.92(\mathrm{~d}, J=7.0 \mathrm{~Hz}, 1 \mathrm{H}, \mathrm{ArH})$, $7.85(\mathrm{~d}, J=7.5 \mathrm{~Hz}, 1 \mathrm{H}, \mathrm{ArH}), 7.56 \sim 7.61(\mathrm{~m}, 3 \mathrm{H}, \mathrm{ArH})$, $7.40 \sim 7.46(\mathrm{~m}, 1 \mathrm{H}, \mathrm{ArH}), 7.23(\mathrm{~d}, J=8.0 \mathrm{~Hz}, 2 \mathrm{H}, \operatorname{ArH})$, $7.14 \sim 7.17$ (dd, $J=2.5,6.5 \mathrm{~Hz}, 2 \mathrm{H}, \mathrm{ArH}), 7.08$ (t, $J=7.5$ $\mathrm{Hz}, 1 \mathrm{H}, \mathrm{ArH}), 6.96$ (s, 1H, 3-H), $6.88 \sim 6.94(\mathrm{~m}, 2 \mathrm{H}, \mathrm{ArH})$, $4.53 \sim 4.57$ (m, 4H, $\mathrm{CH}_{2} \mathrm{CH}_{2}$ ); ESI-MS m/z: 157.14 (100), 179.14 (91), 217.33 (46), 237.15 (39), 261.32 (51), 521.25 $\left([\mathrm{M}+\mathrm{H}]^{+}, 29\right), 543.31\left([\mathrm{M}+\mathrm{Na}]^{+}, 20\right)$.

7-(2-对羟基苯甲酰腙基)-苯氧乙氧基黄酮 $(4 \mathrm{~g})$ ：白 色固体, 产率 75.8\%. m.p. $198 \sim 200{ }^{\circ} \mathrm{C} ;{ }^{1} \mathrm{H}$ NMR (DMSO- $\left.d_{6}, 500 \mathrm{MHz}\right) \delta: 11.65(\mathrm{~s}, 1 \mathrm{H}, \mathrm{NH}), 10.02(\mathrm{~s}, 1 \mathrm{H}$, $\mathrm{OH}), 8.78(\mathrm{~s}, 1 \mathrm{H}, \mathrm{CH}=\mathrm{N}), 8.08 \sim 8.10(\mathrm{~m}, 2 \mathrm{H}, \mathrm{ArH}), 7.97$ (d, $J=9.0 \mathrm{~Hz}, 1 \mathrm{H}, \mathrm{ArH}), 7.89$ (s, 1H, ArH), 7.77 (d, $J=8.5$ $\mathrm{Hz}, 2 \mathrm{H}, \mathrm{ArH}), 7.56 \sim 7.63$ (m, 3H, ArH), $7.41 \sim 7.44(\mathrm{~m}$, 2H, ArH), 7.21 (d, $J=8.5 \mathrm{~Hz}, 1 \mathrm{H}, \mathrm{ArH}), 7.15 \sim 7.17(\mathrm{~m}$, 1H, ArH), 7.07 (t, $J=7.5 \mathrm{~Hz}, 1 \mathrm{H}, \mathrm{ArH}), 6.97$ (s, 1H, 3-H), $6.81(\mathrm{~d}, J=8.5 \mathrm{~Hz}, 2 \mathrm{H}, \mathrm{ArH}), 4.51 \sim 4.56\left(\mathrm{~m}, 4 \mathrm{H}, \mathrm{CH}_{2-}\right.$ $\mathrm{CH}_{2}$ ); ESI-MS $m / z$ : 157.14 (100), 179.33 (88), 217.20 (68), 239.39 (70), 261.25 (77), $521.32\left([\mathrm{M}+\mathrm{H}]^{+}, 78\right), 543.38$ $\left([\mathrm{M}+\mathrm{Na}]^{+}, 59\right)$.

7-(2-对氯苯甲酰腙基)-苯氧乙氧基黄酮 $(\mathbf{4 h})$ : 白色 固体, 产率 83.2\%. m.p. $252 \sim 254{ }^{\circ} \mathrm{C} ;{ }^{1} \mathrm{H}$ NMR $\left(\right.$ DMSO- $\left._{6}, 500 \mathrm{MHz}\right) \delta: 11.91(\mathrm{~s}, 1 \mathrm{H}, \mathrm{NH}), 8.80(\mathrm{~s}, 1 \mathrm{H}$, $\mathrm{CH}=\mathrm{N}), 8.08 \sim 8.09(\mathrm{~m}, 2 \mathrm{H}, \mathrm{ArH}), 7.97(\mathrm{~d}, J=9.0 \mathrm{~Hz}$, 1H, ArH), 7.90 (d, $J=8.0 \mathrm{~Hz}, 3 \mathrm{H}, \mathrm{ArH}), 7.54 \sim 7.61(\mathrm{~m}$, $5 \mathrm{H}, \mathrm{ArH}), 7.42 \sim 7.45(\mathrm{~m}, 2 \mathrm{H}, \mathrm{ArH}), 7.22(\mathrm{~d}, J=8.5 \mathrm{~Hz}$, $1 \mathrm{H}, \operatorname{ArH}), 7.16(\mathrm{~d}, J=2.5 \mathrm{~Hz}, 1 \mathrm{H}, \operatorname{ArH}), 7.14(\mathrm{~m}, 1 \mathrm{H}$, ArH), 6.96 (s, 1H, 3-H), 4.52 4.56 (m, 4H, $\left.\mathrm{CH}_{2} \mathrm{CH}_{2}\right)$; IR (KBr) v: 3274, 3067, 1667, 1599, 1485, 1444, 1376, 1285, 1246, 1164, 1097, 1012, 843, 741, $684 \mathrm{~cm}^{-1}$; ESI-MS $\mathrm{m} / z$ : 159.13 (37), 338.63 (35), 407.91 (34), $539.36\left([\mathrm{M}+\mathrm{H}]^{+}\right.$, 74), $561.31\left([\mathrm{M}+\mathrm{Na}]^{+}, 100\right)$; HRMS calcd for $\mathrm{C}_{31} \mathrm{H}_{24} \mathrm{ClN}_{2} \mathrm{O}_{5}[\mathrm{M}+\mathrm{H}]^{+}$539.1368, found 539.1366.

7-(2-对硝基苯甲酰腙基)-苯氧乙氧基黄酮(4i)：黄 色固体, 产率 $86.7 \%$. m.p. $263 \sim 265{ }^{\circ} \mathrm{C} ;{ }^{1} \mathrm{H}$ NMR (DMSO- $\left.d_{6}, 500 \mathrm{MHz}\right) \delta: 12.12(\mathrm{~s}, 1 \mathrm{H}, \mathrm{NH}), 8.82(\mathrm{~s}, 1 \mathrm{H}$, $\mathrm{CH}=\mathrm{N}), 8.31(\mathrm{~d}, J=8.5 \mathrm{~Hz}, 2 \mathrm{H}, \mathrm{ArH}), 8.11(\mathrm{~d}, J=8.5$ $\mathrm{Hz}, 2 \mathrm{H}, \mathrm{ArH}), 8.08$ (d, $J=6.5 \mathrm{~Hz}, 2 \mathrm{H}, \mathrm{ArH}), 7.97$ (d, $J=$ $9.0 \mathrm{~Hz}, 1 \mathrm{H}, \mathrm{ArH}), 7.93(\mathrm{~d}, J=6.5 \mathrm{~Hz}, 1 \mathrm{H}, \operatorname{ArH}), 7.56 \sim$ 7.61 (m, 3H, ArH), 7.47 (t, J=7.25 Hz, 1H, ArH), 7.42 (d, $J=2.0 \mathrm{~Hz}, 1 \mathrm{H}, \operatorname{ArH}), 7.24(\mathrm{~d}, J=8.5 \mathrm{~Hz}, 1 \mathrm{H}, \operatorname{ArH})$, $7.14 \sim 7.16$ (dd, $J=2.5,6.5 \mathrm{~Hz}, 1 \mathrm{H}, \mathrm{ArH}), 7.09$ (t, $J=7.5$ 
$\mathrm{Hz}, 1 \mathrm{H}, \mathrm{ArH}), 6.96$ (s, 1H, 3-H), 4.53 4.57 (m, 4H, $\mathrm{CH}_{2} \mathrm{CH}_{2}$ ); IR (KBr) v: 3278, 3079, 2936, 2870, 1669, $1630,1606,1561,1525,1488,1447,1379,1347,1284$, 1245, 1166, 1102, 841, 776, 688, $611 \mathrm{~cm}^{-1}$; ESI-MS m/z: 159.07 (68), 338.50 (95), 360.51 (88), 408.24 (70), 572.51 $\left([\mathrm{M}+\mathrm{Na}]^{+}, 100\right)$; HRMS calcd for $\mathrm{C}_{31} \mathrm{H}_{24} \mathrm{~N}_{3} \mathrm{O}_{7}[\mathrm{M}+\mathrm{H}]^{+}$ 550.1609 , found 550.1615 .

7-(2-苯乙酰腙基)-苯氧乙氧基黄酮 $(\mathbf{4 j})$ : 白色固体, 产率 59.5\%. m.p. $148 \sim 150{ }^{\circ} \mathrm{C} ;{ }^{1} \mathrm{H}$ NMR (DMSO- $d_{6}, 500$ $\mathrm{MHz}) \delta: 11.61(\mathrm{~s}, 0.42 \mathrm{H}), 11.33(\mathrm{~s}, 0.56 \mathrm{H})(\mathrm{NH}), 8.56(\mathrm{~s}$, $0.41 \mathrm{H}), 8.38(\mathrm{~s}, 0.56 \mathrm{H})(\mathrm{CH}=\mathrm{N}), 8.08 \sim 8.11(\mathrm{~m}, 2 \mathrm{H}$, $\operatorname{ArH}), 7.98$ (t, $J=8.68 \mathrm{~Hz}, 1 \mathrm{H}, 5-\mathrm{H}), 7.81 \sim 7.87$ (m, 1H, ArH), $7.59 \sim 7.60(\mathrm{~m}, 3 \mathrm{H}, \mathrm{ArH}, 8-\mathrm{H}), 7.38 \sim 7.43(\mathrm{~m}, 2 \mathrm{H}$, ArH), $7.27 \sim 7.30$ (m, 4H, ArH), $7.01 \sim 7.18$ (m, 4H, ArH), $6.98(\mathrm{~s}, 1 \mathrm{H}, 3-\mathrm{H}), 4.50 \sim 4.55\left(\mathrm{~m}, 4 \mathrm{H}, \mathrm{CH}_{2} \mathrm{CH}_{2}\right), 3.94(\mathrm{~s}$, $0.87 \mathrm{H}), 3.49(\mathrm{~s}, 1.13 \mathrm{H})\left(\mathrm{CH}_{2}\right)$; HRMS calcd for $\mathrm{C}_{32} \mathrm{H}_{27^{-}}$ $\mathrm{N}_{2} \mathrm{O}_{5}[\mathrm{M}+\mathrm{H}]^{+}$519.1914, found 519.1929.

7-(2-对硝基苯乙酰腙基)-苯氧乙氧基黄酮(4k): 白 色固体，产率 63.9\%. m.p. 250 252 ${ }^{\circ} \mathrm{C} ;{ }^{1} \mathrm{H} \mathrm{NMR}$ $\left(\mathrm{DMSO}-d_{6}, 400 \mathrm{MHz}\right) \delta: 11.69(\mathrm{~s}, 0.35 \mathrm{H}), 11.45(\mathrm{~s}, 0.60 \mathrm{H})$ $(\mathrm{NH}), 8.55(\mathrm{~s}, 0.32 \mathrm{H}), 8.37(\mathrm{~s}, 0.56 \mathrm{H})(\mathrm{CH}=\mathrm{N}), 8.07 \sim$ 8.19 (m, 4H, ArH, 5-H), $7.94 \sim 7.99$ (m, 1H, ArH), 7.80 $7.85(\mathrm{~m}, 1 \mathrm{H}, \mathrm{ArH}), 7.51 \sim 7.60(\mathrm{~m}, 5 \mathrm{H}, \mathrm{ArH}, 8-\mathrm{H}), 7.37 \sim$ $7.44(\mathrm{~m}, 2 \mathrm{H}, \mathrm{ArH}), 7.11 \sim 7.21(\mathrm{~m}, 2 \mathrm{H}, \mathrm{ArH}, 6-\mathrm{H}), 6.98 \sim$ $7.07(\mathrm{~m}, 1 \mathrm{H}, \mathrm{ArH}), 6.95(\mathrm{~s}, 1 \mathrm{H}, 3-\mathrm{H}), 4.50 \sim 4.56(\mathrm{~m}, 4 \mathrm{H}$, $\left.\mathrm{CH}_{2} \mathrm{CH}_{2}\right), 4.12(\mathrm{~s}, 0.71 \mathrm{H}), 3.67(\mathrm{~s}, 1.16 \mathrm{H})\left(\mathrm{CH}_{2}\right)$; HRMS calcd for $\mathrm{C}_{32} \mathrm{H}_{26} \mathrm{~N}_{3} \mathrm{O}_{7} \quad[\mathrm{M}+\mathrm{H}]{ }^{+}$564.1765, found 564.1782 .

7-(2- $\alpha$-菜乙酰腙基)-苯氧乙氧基黄酮(4I): 白色固 体, 产率 90.2\%. m.p. 178 $181{ }^{\circ} \mathrm{C} ;{ }^{1} \mathrm{H}$ NMR (DMSO- $d_{6}$, $400 \mathrm{MHz}) \delta: 11.74(\mathrm{~s}, 0.40 \mathrm{H}), 11.42(\mathrm{~s}, 0.60 \mathrm{H})(\mathrm{NH}), 8.59$ $(\mathrm{s}, 0.40 \mathrm{H}), 8.42(\mathrm{~s}, 0.60 \mathrm{H})(\mathrm{CH}=\mathrm{N}), 8.07 \sim 8.12(\mathrm{~m}, 2 \mathrm{H}$, ArH, 5-H), $7.98 \sim 8.03(\mathrm{~m}, 1 \mathrm{H}, \mathrm{ArH}), 7.77 \sim 7.93(\mathrm{~m}, 4 \mathrm{H}$, $\mathrm{ArH}), 7.57 \sim 7.60(\mathrm{~m}, 3 \mathrm{H}, \mathrm{ArH}), 7.40 \sim 7.54(\mathrm{~m}, 6 \mathrm{H}, \mathrm{ArH}$, $8-\mathrm{H}), 7.11 \sim 7.22(\mathrm{~m}, 2 \mathrm{H}, \mathrm{ArH}, 6-\mathrm{H}), 7.00 \sim 7.06(\mathrm{~m}, 1 \mathrm{H}$, $\operatorname{ArH}), 6.96(\mathrm{~s}, 1 \mathrm{H}, 3-\mathrm{H}), 4.51 \sim 4.57\left(\mathrm{~m}, 4 \mathrm{H}, \mathrm{CH}_{2} \mathrm{CH}_{2}\right)$, $4.41(\mathrm{~s}, 1.27 \mathrm{H}), 3.99(\mathrm{~s}, 0.81 \mathrm{H})\left(\mathrm{CH}_{2}\right)$; IR $(\mathrm{KBr}) v: 3241$, 3208, 3062, 2948, 2884, 1672, 1655, 1627, 1606, 1545, $1490,1448,1371,1290,1249,1165,1148,1095,1071$, 1044, 962, 927, 877, 778, 754, 688, $545 \mathrm{~cm}^{-1}$; ESI-MS $m / z: 569.3\left([\mathrm{M}+\mathrm{H}]^{+}, 100\right)$.

7-(2-苯氧乙酰腙基)-苯氧乙氧基黄酮(4m): 白色固 体, 产率 93.4\%. m.p. 187 $189{ }^{\circ} \mathrm{C} ;{ }^{1} \mathrm{H}$ NMR (DMSO- $d_{6}$, $400 \mathrm{MHz}) \delta: 11.67(\mathrm{~s}, 0.38 \mathrm{H}), 11.57(\mathrm{~s}, 0.53 \mathrm{H})(\mathrm{NH}), 8.65$ $(\mathrm{s}, 0.35 \mathrm{H}), 8.38(\mathrm{~s}, 0.57 \mathrm{H})(\mathrm{CH}=\mathrm{N}), 8.11(\mathrm{~d}, J=7.36 \mathrm{~Hz}$,
2H, ArH), 7.96 7.99 (dd, $J=2.56,6.28 \mathrm{~Hz}, 1 \mathrm{H}, \mathrm{ArH})$, $7.85(\mathrm{t}, J=7.72 \mathrm{~Hz}, 1 \mathrm{H}, \mathrm{ArH}), 7.59 \sim 7.62(\mathrm{~m}, 3 \mathrm{H}, \mathrm{ArH})$, $7.42 \sim 7.45(\mathrm{~m}, 2 \mathrm{H}, \mathrm{ArH}), 7.20 \sim 7.31(\mathrm{~m}, 3 \mathrm{H}, \mathrm{ArH})$, $7.14 \sim 7.17(\mathrm{dd}, J=2.04,6.76 \mathrm{~Hz}, 1 \mathrm{H}, \operatorname{ArH}), 7.05$ (t, $J=$ $7.48 \mathrm{~Hz}, 1 \mathrm{H}, \mathrm{ArH}), 7.00$ (s, 1H, 3-H), 6.87 $6.96(\mathrm{~m}, 3 \mathrm{H}$, $\mathrm{ArH}), 4.60(\mathrm{~s}, 1.08 \mathrm{H}), 4.54(\mathrm{~s}, 0.87 \mathrm{H})\left(\mathrm{CH}_{2}\right), 4.51 \sim 4.53$ $\left(\mathrm{m}, 4 \mathrm{H}, \mathrm{CH}_{2} \mathrm{CH}_{2}\right)$; IR (KBr) v: 3456, 3222, 3074, 2947, 2884, 1687, 1632, 1555, 1500, 1448, 1377, 1242, 1171, 1094, 839, 760, $683 \mathrm{~cm}^{-1}$; ESI-MS m/z: $535.0\left([\mathrm{M}+\mathrm{H}]^{+}\right.$, 100); HRMS calcd for $\mathrm{C}_{32} \mathrm{H}_{27} \mathrm{~N}_{2} \mathrm{O}_{6}[\mathrm{M}+\mathrm{H}]^{+}$535.1864, found 535.1879 .

7-(2,4-二氯苯氧乙酰腙基)-苯氧乙氧基黄酮 $(4 n)$ : 白色固体，产率 93.7\%. m.p. 221 223 ${ }^{\circ} \mathrm{C} ;{ }^{1} \mathrm{H}$ NMR (DMSO- $\left.d_{6}, 400 \mathrm{MHz}\right) \delta: 11.75(\mathrm{~s}, 0.29 \mathrm{H}), 11.72(\mathrm{~s}, 0.69 \mathrm{H})$ $(\mathrm{NH}), 8.57(\mathrm{~s}, 0.24 \mathrm{H}), 8.35(\mathrm{~s}, 0.74 \mathrm{H})(\mathrm{CH}=\mathrm{N}), 8.09 \sim$ $8.11(\mathrm{~m}, 2 \mathrm{H}, \mathrm{ArH}), 7.94 \sim 7.99(\mathrm{~m}, 1 \mathrm{H}, \operatorname{ArH}), 7.84$ (d, $J=$ $7.72 \mathrm{~Hz}, 1 \mathrm{H}, \mathrm{ArH}), 7.57 \sim 7.76(\mathrm{~m}, 3 \mathrm{H}, \mathrm{ArH}), 7.52$ (d, $J=$ $2.52 \mathrm{~Hz}, 1 \mathrm{H}, \mathrm{ArH}), 7.40 \sim 7.44(\mathrm{~m}, 2 \mathrm{H}, \mathrm{ArH}), 7.27 \sim 7.30$ $(\mathrm{dd}, J=2.52,6.40 \mathrm{~Hz}, 1 \mathrm{H}, \mathrm{ArH}), 7.13 \sim 7.22(\mathrm{~m}, 2 \mathrm{H}$, ArH), $6.98 \sim 7.07$ (m, 3H, ArH, 3-H), $5.24(\mathrm{~s}, 1.45 \mathrm{H}), 4.74$ $(\mathrm{s}, 0.52 \mathrm{H})\left(\mathrm{CH}_{2}\right), 4.51 \sim 4.74\left(\mathrm{~m}, 4 \mathrm{H}, \mathrm{CH}_{2} \mathrm{CH}_{2}\right)$; IR $(\mathrm{KBr})$ v: 3448, 3223, 3082, 2939, 1703, 1632, 1483, 1448, 1377, 1250, 1165, 1094, 845, 761, $683 \mathrm{~cm}^{-1}$; ESI-MS m/z: 274.1 (100), 318.2 (40), 435.9 (32), $603.0\left(\mathrm{M}^{+}, 64\right)$; HRMS calcd for $\mathrm{C}_{32} \mathrm{H}_{25} \mathrm{Cl}_{2} \mathrm{~N}_{2} \mathrm{O}_{6} \quad[\mathrm{M}+\mathrm{H}]^{+}$603.1084, found 603.1098.

3.2 .4 含 $1,3,4$-噁二唑(啉)苯氧乙氧基黄酮衍生物的 合成

以 $5 \mathbf{a}$ 的合成为例: 将 $1.0 \mathrm{mmol}$ 7-(2-苯甲酰腙基)苯氧乙氧基黄酮(4a)置于 $50 \mathrm{~mL}$ 圆底烧瓶中, 加入 5 $\mathrm{mL}$ 乙酸酐, 控制温度在 $100{ }^{\circ} \mathrm{C}$ 左右, TLC 跟踪反应至 原料消失. 停止反应, 将反应液冷至室温, 然后慢慢倒 入冰水中, 剧烈搅拌至油状物固化, 过滤, 干燥, 得粗 产品, 无水乙醇重结晶得目标产物 5a. 类似方法得到其 它化合物.

7-[2-(5-苯基-1,3,4-噁二唑啉)]-苯氧乙氧基黄酮 (5a): 白色固体，产率 47.3\%. m.p. $213 \sim 215{ }^{\circ} \mathrm{C} ;{ }^{1} \mathrm{H}$ NMR (DMSO- $\left.d_{6}, 500 \mathrm{MHz}\right) \delta: 8.06 \sim 8.08(\mathrm{dd}, J=1.8$, $6.28 \mathrm{~Hz}, 2 \mathrm{H}, \mathrm{ArH}), 7.91(\mathrm{~d}, J=8.84 \mathrm{~Hz}, 1 \mathrm{H}, \mathrm{ArH}), 7.56 \sim$ $7.61(\mathrm{~m}, 5 \mathrm{H}, \mathrm{ArH}), 7.29 \sim 7.36(\mathrm{~m}, 5 \mathrm{H}, \mathrm{ArH}), 7.16 \sim 7.21$ (m, 3H, ArH, OCHN), 7.01 (t, $J=7.48 \mathrm{~Hz}, 1 \mathrm{H}, \mathrm{ArH})$, $6.94 \sim 6.97(\mathrm{dd}, J=2.28,6.52 \mathrm{~Hz}, 1 \mathrm{H}, \mathrm{ArH}), 6.93(\mathrm{~s}, 1 \mathrm{H}$, $3-\mathrm{H}), 4.39 \sim 4.42\left(\mathrm{~m}, 4 \mathrm{H}, \mathrm{CH}_{2} \mathrm{CH}_{2}\right), 2.19\left(\mathrm{~s}, 3 \mathrm{H}, \mathrm{CH}_{3}\right) ;{ }^{13} \mathrm{C}$ NMR (125 MHz, DMSO- $\left.d_{6}\right) \delta: 175.88,162.64,161.93$, $157.04,156.46,154.20,131.13,131.02,131.00,130.93$, $128.66,128.40,128.30,125.96,125.82,125.78,124.00$, 
$123.91,120.51,117.10,114.47,112.91,106.55,101.52$, $89.57,66.89,66.91,40.42,40.02,39.85,39.68,39.52$, 20.69; IR (KBr) v: 3067, 2939, 2883, 1647, 1602, 1575, 1497, 1374, 1277, 1177, 1088, 847, 771, $689 \mathrm{~cm}^{-1}$; ESIMS $m / z$ : 104.9 (16), 274.2 (16), 505.0 (57) , 547.0 ([M+ $\left.\mathrm{H}]^{+}, 100\right)$; HRMS calcd for $\mathrm{C}_{33} \mathrm{H}_{27} \mathrm{~N}_{2} \mathrm{O}_{6}[\mathrm{M}+\mathrm{H}]^{+}$ 547.1864, found 547.1840.

7-[2-(5-邻甲苯基-1,3,4-噁二唑)]-苯氧乙氧基黄酮 (5b): 白色固体, 产率 32.6\%. m.p. 199 201 ${ }^{\circ} \mathrm{C} ;{ }^{1} \mathrm{H}$ NMR (DMSO- $\left.d_{6}, 500 \mathrm{MHz}\right) \delta: 8.07 \sim 8.09$ (dd, $J=1.5$, $6.0 \mathrm{~Hz}, 2 \mathrm{H}, \mathrm{ArH}), 8.02 \sim 8.04(\mathrm{dd}, J=1.5,6.5 \mathrm{~Hz}, 1 \mathrm{H}$, ArH), 7.89 (d, $J=8.5 \mathrm{~Hz}, 1 \mathrm{H}, \operatorname{ArH}), 7.82(\mathrm{~d}, J=7.5 \mathrm{~Hz}$, $1 \mathrm{H}, \operatorname{ArH}), 7.68(\mathrm{t}, J=7.0 \mathrm{~Hz}, 1 \mathrm{H}, \mathrm{ArH}), 7.58 \sim 7.65(\mathrm{~m}$, 4H, ArH), 7.40 (d, $J=8.5 \mathrm{~Hz}, 1 \mathrm{H}, \operatorname{ArH}), 7.33$ (d, $J=2.5$ $\mathrm{Hz}, 1 \mathrm{H}, \operatorname{ArH}), 7.29 \sim 7.31(\mathrm{~m}, 2 \mathrm{H}, \operatorname{ArH}), 7.21$ (t, $J=7.5$ $\mathrm{Hz}, 1 \mathrm{H}, \mathrm{ArH}), 7.00 \sim 7.05$ (m, 2H, ArH), 6.95 (s, 1H, 3-H), $4.59\left(\mathrm{~s}, 4 \mathrm{H}, \mathrm{CH}_{2} \mathrm{CH}_{2}\right), 2.59$ (s, 3H, $\left.\mathrm{CH}_{3}\right) ;{ }^{13} \mathrm{C}$ NMR $(125$ MHz, DMSO- $d_{6}$ ) $\delta: 175.85,163.97,162.61,162.22$, $161.93,157.01,156.31,137.15,133.09,131.13,131.02$, $130.62,129.88,128.64,128.14,125.91,125.82,125.68$, $122.31,121.07,117.15,114.28,114.17,112.86,106.57$, $101.60,67.39,67.26,40.02,39.85,39.68,20.77$; IR (KBr) $v: 3067,2955,2883,1639,1603,1539,1448,1369,1250$, $1171,1043,831,768,683 \mathrm{~cm}^{-1}$; ESI-MS $\mathrm{m} / z$ : 157.14 (100), $179.14(56), 517.30\left([\mathrm{M}+\mathrm{H}]^{+}, 86\right), 539.30([\mathrm{M}+$ $\mathrm{Na}]^{+}$, 17) ; HRMS calcd for $\mathrm{C}_{32} \mathrm{H}_{25} \mathrm{~N}_{2} \mathrm{O}_{5}[\mathrm{M}+\mathrm{H}]^{+}$ 517.5443, found 517.5441.

7-[2-(4-乙酰基-5-间甲苯基-1,3,4-噁二唑啉)]-苯氧 乙氧基黄酮 $(\mathbf{5 c})$ : 淡黄色固体, 产率 73.1\%. m.p. 205 $207{ }^{\circ} \mathrm{C} ;{ }^{1} \mathrm{H}$ NMR (DMSO- $\left.d_{6}, 500 \mathrm{MHz}\right) \delta: 8.07 \sim 8.09$ (dd, $J=1.5,7.0 \mathrm{~Hz}, 2 \mathrm{H}, \mathrm{ArH}), 7.90(\mathrm{~d}, J=9.0 \mathrm{~Hz}, 1 \mathrm{H}$, ArH), $7.56 \sim 7.64(\mathrm{~m}, 2 \mathrm{H}, \mathrm{ArH}), 7.42 \sim 7.46(\mathrm{~m}, 3 \mathrm{H}, \mathrm{ArH})$, $7.37 \sim 7.38$ (dd, $J=1.5,6.5 \mathrm{~Hz}, 1 \mathrm{H}, \mathrm{ArH}), 7.14 \sim 7.17(\mathrm{~m}$, $1 \mathrm{H}, \mathrm{ArH}), 7.02 \sim 7.05(\mathrm{~m}, 3 \mathrm{H}, \mathrm{ArH}, \mathrm{OCHN}), 6.95(\mathrm{~s}, 1 \mathrm{H}$, $3-\mathrm{H}), 6.92 \sim 6.94(\mathrm{~m}, 4 \mathrm{H}, \mathrm{ArH}), 4.32 \sim 4.34(\mathrm{~m}, 4 \mathrm{H}$, $\mathrm{CH}_{2} \mathrm{CH}_{2}$ ), 2.18 (s, 3H, $\mathrm{CH}_{3}$ ), 2.13 (s, 3H, $\left.\mathrm{CH}_{3}\right) ;{ }^{13} \mathrm{C} \mathrm{NMR}$ $\left(125 \mathrm{MHz}, \mathrm{DMSO}-d_{6}\right) \delta: 175.84,162.59,161.93,157.02$, $156.50,152.58,152.44,131.11,131.04,131.02,129.78$, $128.67,128.63,127.31,125.99,125.81,125.78,123.80$, $120.43,117.09,114.06,112.80,106.53,101.44,89.96$, $67.33,66.90,40.02,39.85,39.68,39.52,39.35,20.69$, 20.21; IR (KBr) v: 3074, 2933, 2876, 1759, 1639, 1603, 1497, 1448, 1369, 1250, 1192, 1065, 908, 853, 760, 683 $\mathrm{cm}^{-1}$; ESI-MS m/z: $157.14(100), 561.42\left([\mathrm{M}+\mathrm{H}]^{+}, 69\right)$, $583.29\left([\mathrm{M}+\mathrm{Na}]^{+}, 15\right)$; HRMS calcd for $\mathrm{C}_{34} \mathrm{H}_{29} \mathrm{~N}_{2} \mathrm{O}_{6}$
$[\mathrm{M}+\mathrm{H}]^{+}$561.5967, found 561.5960.

7-[2-(4-乙酰基-5-对甲苯基-1,3,4-噁二唑啉)]一苯氧 乙氧基黄酮 $(5 d)$ : 白色固体，产率 46.8\%. m.p. 173 $175{ }^{\circ} \mathrm{C} ;{ }^{1} \mathrm{H}$ NMR (DMSO-d $\left.6,500 \mathrm{MHz}\right) \delta: 8.09 \sim 8.11$ $(\mathrm{dd}, J=1.5,6.0 \mathrm{~Hz}, 2 \mathrm{H}, \mathrm{ArH}), 8.01 \sim 8.03(\mathrm{dd}, J=2.0,6.0$ $\mathrm{Hz}, 1 \mathrm{H}, \mathrm{ArH}), 7.91$ (d, $J=8.5 \mathrm{~Hz}, 1 \mathrm{H}, \mathrm{ArH}), 7.74$ (d, $J=$ $8.5 \mathrm{~Hz}, 1 \mathrm{H}, \mathrm{ArH}), 7.58 \sim 7.66(\mathrm{~m}, 4 \mathrm{H}, \mathrm{ArH}), 7.38 \sim 7.47$ $(\mathrm{m}, 3 \mathrm{H}, \operatorname{ArH}), 7.18 \sim 7.21(\mathrm{~m}, 1 \mathrm{H}, \operatorname{ArH}), 7.12(\mathrm{~s}, 1 \mathrm{H}$, OCHN), $6.98 \sim 7.08(\mathrm{~m}, 3 \mathrm{H}, \mathrm{ArH}), 6.95(\mathrm{~s}, 1 \mathrm{H}, 3-\mathrm{H})$, $4.40 \sim 4.62\left(\mathrm{~m}, 4 \mathrm{H}, \mathrm{CH}_{2} \mathrm{CH}_{2}\right), 2.18\left(\mathrm{~s}, 3 \mathrm{H}, \mathrm{CH}_{3}\right), 2.15(\mathrm{~s}$, $\left.3 \mathrm{H}, \mathrm{CH}_{3}\right) ;{ }^{13} \mathrm{C}$ NMR (125 MHz, DMSO- $\left.d_{6}\right) \delta: 175.84$, $162.46,161.94,157.06,156.49,156.26,141.27,133.05$, $131.14,131.01,129.76,129.18,128.64,128.56,125.85$, $125.80,125.76,125.73,120.40,117.17,114.32,112.82$, $106.53,101.46,89.59,67.34,66.91,40.02,39.85,39.68$, 39.52 , 39.35, 20.69, 20.51; IR (KBr) v: 3074, 2926, 2883, 1639, 1610, 1504, 1448, 1377, 1249, 1170, 1080, 831, 760, $675 \mathrm{~cm}^{-1}$; ESI-MS m/z: 157.14 (100), 179.14 (90), 517.30 (65), $561.48\left([\mathrm{M}+\mathrm{H}]^{+}, 11\right)$; HRMS calcd for $\mathrm{C}_{34} \mathrm{H}_{29} \mathrm{~N}_{2} \mathrm{O}_{6}$ $[\mathrm{M}+\mathrm{H}]^{+}$561.5967, found 561.5973.

7-[2-(4-乙酰基-5-对甲氧苯基-1,3,4-噁二唑啉)]-苯 氧乙氧基黄酮(5e): 白色固体, 产率 72.0\%. m.p. 237 $239{ }^{\circ} \mathrm{C} ;{ }^{1} \mathrm{H}$ NMR (DMSO- $\left.d_{6}, 500 \mathrm{MHz}\right) \delta: 8.06 \sim 8.07(\mathrm{~m}$, $2 \mathrm{H}, \operatorname{ArH}), 7.90(\mathrm{~d}, J=8.5 \mathrm{~Hz}, 1 \mathrm{H}, \operatorname{ArH}), 7.56 \sim 7.61(\mathrm{~m}$, $3 \mathrm{H}, \mathrm{ArH}), 7.51$ (d, $J=8.5 \mathrm{~Hz}, 2 \mathrm{H}, \mathrm{ArH}), 7.43$ (t, $J=8.5$ $\mathrm{Hz}, 1 \mathrm{H}, \mathrm{ArH}), 7.35$ (d, J=7.5 Hz, 1H, ArH), 7.16 7.18 (m, 2H, ArH), 7.11 (s, 1H, OCHN), 7.01 (t, $J=7.5 \mathrm{~Hz}, 1 \mathrm{H}$, $\operatorname{ArH}), 6.96 \sim 6.97(\mathrm{~m}, 1 \mathrm{H}, \mathrm{ArH}), 6.95(\mathrm{~s}, 1 \mathrm{H}, 3-\mathrm{H}), 6.78(\mathrm{~d}$, $J=8.5 \mathrm{~Hz}, 2 \mathrm{H}, \mathrm{ArH}), 4.38 \sim 4.41\left(\mathrm{~m}, 4 \mathrm{H}, \mathrm{CH}_{2} \mathrm{CH}_{2}\right), 3.63$ $\left(\mathrm{s}, 3 \mathrm{H}, \mathrm{CH}_{3}\right), 2.17\left(\mathrm{~s}, 3 \mathrm{H}, \mathrm{CH}_{3}\right) ;{ }^{13} \mathrm{C} \mathrm{NMR}(125 \mathrm{MHz}$, DMSO- $\left.d_{6}\right) \delta: 175.72,162.50,161.93,156.90,156.61$, $152.99,148.20,131.24,131.11,131.03,129.76,128.63$, $128.50,127.73,125.78,125.61,123.32,123.22,120.43$, $117.00,114.42,113.86,106.53,101.49,90.89,66.95$, $66.83,54.91,40.42,40.02,39.85,39.68,39.52,20.72$; IR (KBr) v: 3070, 2939, 2833, 1653, 1610, 1504, 1448, 1369, 1250, 1178, 1080, 1030, 972, 839, 760, $675 \mathrm{~cm}^{-1}$; ESI-MS $m / z: 157.14$ (100), 179.14 (45), $577.36\left([\mathrm{M}+\mathrm{H}]^{+}, 33\right)$, $599.35\left([\mathrm{M}+\mathrm{Na}]^{+}, 8\right)$; HRMS calcd for $\mathrm{C}_{34} \mathrm{H}_{29} \mathrm{~N}_{2} \mathrm{O}_{7}$ $[\mathrm{M}+\mathrm{H}]^{+}$577.5961, found 577.5956.

7-[2-(4-乙酰基-5-邻乙酰氧苯基-1,3,4-啞二唑啉)]苯氧乙氧基黄酮 $(\mathbf{5 f})$ : 淡黄色固体, 产率 42.4\%. m.p. $168 \sim 170{ }^{\circ} \mathrm{C} ;{ }^{1} \mathrm{H}$ NMR (DMSO- $\left.d_{6}, 500 \mathrm{MHz}\right) \delta: 8.06 \sim$ $8.08(\mathrm{dd}, J=1.5,6.5 \mathrm{~Hz}, 2 \mathrm{H}, \operatorname{ArH}), 7.91$ (d, $J=8.5 \mathrm{~Hz}$, $1 \mathrm{H}, \mathrm{ArH}), 7.58 \sim 7.64(\mathrm{~m}, 4 \mathrm{H}, \mathrm{ArH}), 7.44 \sim 7.49(\mathrm{~m}, 3 \mathrm{H}$, 
ArH), $7.25 \sim 7.31(\mathrm{~m}, 2 \mathrm{H}, \mathrm{ArH}), 7.19(\mathrm{~d}, \quad J=8.0 \mathrm{~Hz}, 1 \mathrm{H}$, ArH), $7.12 \sim 7.15(\mathrm{~m}, 2 \mathrm{H}, \mathrm{ArH}, \mathrm{OCHN}), 7.02$ (t, $J=7.5$ $\mathrm{Hz}, 1 \mathrm{H}, \mathrm{ArH}), 6.97 \sim 6.99(\mathrm{~m}, 1 \mathrm{H}, \mathrm{ArH}), 6.93(\mathrm{~s}, 1 \mathrm{H}, 3-\mathrm{H})$, $4.43 \sim 4.60$ (m, 4H, $\left.\mathrm{CH}_{2} \mathrm{CH}_{2}\right), 2.17\left(\mathrm{~s}, 3 \mathrm{H}, \mathrm{CH}_{3}\right), 2.14$ (s, $\left.3 \mathrm{H}, \mathrm{CH}_{3}\right) ;{ }^{13} \mathrm{C}$ NMR (125 MHz, DMSO- $\left.d_{6}\right) \delta: 175.87$, 162.60, 161.94, 157.03, 156.41, 151.51, 148.13, 132.02, $131.11,131.03,129.85,128.64,128.54,128.20,125.81$, $125.76,123.77,123.55,120.56,117.11,114.31,112.62$, 106.55, 101.54, 88.51, 66.96, 66.83, 55.60, 40.02, 39.85, $39.69,39.52,39.35,20.75,20.38$; IR (KBr) v: 3082, 2939, 2883, 1759, 1632, 1450, 1448, 1377, 1284, 1200, 1051, 831, 760, $675 \mathrm{~cm}^{-1}$; ESI-MS m/z: 157.20 (100), 179.14 (51), 261.32 (38), $605.35\left([\mathrm{M}+\mathrm{H}]^{+}, 44\right), 627.22([\mathrm{M}+$ $\mathrm{Na}]^{+}$, 39); HRMS calcd for $\mathrm{C}_{35} \mathrm{H}_{29} \mathrm{~N}_{2} \mathrm{O}_{8}[\mathrm{M}+\mathrm{H}]^{+}$ 605.6062, found 605.6071.

7-[2-(4-乙酰基-5-对乙酰氧苯基-1,3,4-噁二唑啉)]苯氧乙氧基黄酮 $(\mathbf{5 g})$ ：淡黄色固体，产率 73.1\%. m.p. 205 207 ${ }^{\circ} \mathrm{C} ;{ }^{1} \mathrm{H}$ NMR (DMSO- $d_{6}, 500 \mathrm{MHz}$ ) $\delta: 8.06 \sim$ 8.07 (dd, $J=1.5,6.5 \mathrm{~Hz}, 2 \mathrm{H}, \mathrm{ArH}), 7.90(\mathrm{~d}, J=8.5 \mathrm{~Hz}$, $1 \mathrm{H}, \mathrm{ArH}), 7.56 \sim 7.61(\mathrm{~m}, 3 \mathrm{H}, \mathrm{ArH}), 7.39 \sim 7.46(\mathrm{~m}, 2 \mathrm{H}$, $\mathrm{ArH}), 7.35 \sim 7.36(\mathrm{~m}, 2 \mathrm{H}, \mathrm{ArH}), 7.15 \sim 7.21(\mathrm{~m}, 5 \mathrm{H}, \mathrm{ArH}$, OCHN), 7.01 (t, $J=7.5 \mathrm{~Hz}, 1 \mathrm{H}, \mathrm{ArH}), 6.94(\mathrm{~d}, J=2.5 \mathrm{~Hz}$, $1 \mathrm{H}, \mathrm{ArH}), 6.93$ (s, 1H, 3-H), 4.40 4.41 (m, 4H, $\mathrm{CH}_{2} \mathrm{CH}_{2}$ ), $2.18\left(\mathrm{~s}, 3 \mathrm{H}, \mathrm{CH}_{3}\right), 2.15\left(\mathrm{~s}, 3 \mathrm{H}, \mathrm{CH}_{3}\right) ;{ }^{13} \mathrm{C} \mathrm{NMR}(125 \mathrm{MHz}$, DMSO- $\left.d_{6}\right) \delta: 175.86,162.64,161.88,157.00,156.47$, 154.26, 137.78, 131.11, 131.00, 130.97, 129.83, 128.63, $128.54,126.24,125.80,125.74,123.89,123.17,120.44$, $117.07,114.17,112.79,106.51,101.44,89.63,66.94$, 66.90, 55.69, 40.42, 40.02, 39.85, 39.68, 39.52, 20.70, 20.21; IR (KBr) v: 3074, 2939, 2876, 1639, 1603, 1497, 1448, 1362, 1242, 1178, 1086, 980, 845, 768, $696 \mathrm{~cm}^{-1}$; ESI-MS $m / z: 179.14$ (99), $605.35\left([\mathrm{M}+\mathrm{H}]^{+}, 100\right), 627.34$ $\left([\mathrm{M}+\mathrm{Na}]^{+}, 19\right)$; HRMS calcd for $\mathrm{C}_{35} \mathrm{H}_{29} \mathrm{~N}_{2} \mathrm{O}_{8}[\mathrm{M}+\mathrm{H}]^{+}$ 605.6062, found 605.6056 .

7-[2-(4-乙酰基-5-对氯苯基-1,3,4-噁二唑啉)]-苯氧 乙氧基黄酮(5h): 白色固体, 产率 77.8\%. m.p. 221 $223{ }^{\circ} \mathrm{C} ;{ }^{1} \mathrm{H}$ NMR (DMSO- $\left.d_{6}, 500 \mathrm{MHz}\right) \delta: 8.07 \sim 8.09$ (m, 2H, ArH), 7.90 (d, $J=9.0 \mathrm{~Hz}, 1 \mathrm{H}, \mathrm{ArH}), 7.56 \sim 7.60(\mathrm{~m}$, $5 \mathrm{H}, \mathrm{ArH}), 7.44(\mathrm{t}, J=8.0 \mathrm{~Hz}, 1 \mathrm{H}, \mathrm{ArH}), 7.39$ (d, $J=7.5$ $\mathrm{Hz}, 1 \mathrm{H}, \mathrm{ArH}), 7.26$ (d, $J=8.5 \mathrm{~Hz}, 2 \mathrm{H}, \mathrm{ArH}), 7.16$ (d, $J=$ $8.5 \mathrm{~Hz}, 1 \mathrm{H}, \mathrm{ArH}), 7.11 \sim 7.13(\mathrm{~m}, 2 \mathrm{H}, \mathrm{ArH}, \mathrm{OCHN}), 7.02$ (t, $J=7.5 \mathrm{~Hz}, 1 \mathrm{H}, \mathrm{ArH}), 6.91(\mathrm{~s}, 1 \mathrm{H}, 3-\mathrm{H}), 6.89 \sim 6.91(\mathrm{~m}$, $1 \mathrm{H}, \mathrm{ArH}), 4.30 \sim 4.38\left(\mathrm{~m}, 4 \mathrm{H}, \mathrm{CH}_{2} \mathrm{CH}_{2}\right), 2.18(\mathrm{~s}, 3 \mathrm{H}$, $\left.\mathrm{CH}_{3}\right) ;{ }^{13} \mathrm{C}$ NMR (125 MHz, DMSO- $\left.d_{6}\right) \delta: 175.88,162.57$, $161.94,156.99,156.55,153.36,131.10,131.07,131.05$,
$129.82,128.90,128.84,128.63,128.44,125.95,125.82$, $125.79,123.62,120.36,117.08,114.30,112.70,106.54$, $101.72,90.37,66.91,66.86,40.42,40.02,39.85,39.68$, 39.52, 20.69; IR (KBr) v: 3066, 2937, 1643, 1602, 1492, 1445, 1359, 1282, 1244, 1174, 1127, 1075, 1023, 970, 835, 764, $667 \mathrm{~cm}^{-1}$; ESI-MS m/z: 159.19 (34), 338.43 (100), $360.64(63), 581.39\left([\mathrm{M}+\mathrm{H}]^{+}\right.$, 37); HRMS calcd for $\mathrm{C}_{33} \mathrm{H}_{26} \mathrm{ClN}_{2} \mathrm{O}_{6}[\mathrm{M}+\mathrm{H}]^{+}$581.1474, found 581.1461.

7-[2-(4-乙酰基-5-对硝基苯基-1,3,4-噁二唑啉)]-苯 氧乙氧基黄酮 $(\mathbf{5 i})$ : 黄色固体, 产率 79.6\%. m.p. 235 $237{ }^{\circ} \mathrm{C} ;{ }^{1} \mathrm{H}$ NMR (DMSO- $d_{6}, 500 \mathrm{MHz}$ ) $\delta: 8.05 \sim 8.07$ (dd, $J=1.0,6.5 \mathrm{~Hz}, 2 \mathrm{H}, \mathrm{ArH}), 7.97 \sim 7.99(\mathrm{~m}, 2 \mathrm{H}, \mathrm{ArH})$, $7.81(\mathrm{t}, J=8.75 \mathrm{~Hz}, 3 \mathrm{H}, \mathrm{ArH}), 7.57 \sim 7.61(\mathrm{~m}, 3 \mathrm{H}, \mathrm{ArH})$, 7.45 (d, $J=7.5 \mathrm{~Hz}, 2 \mathrm{H}, \mathrm{ArH}), 7.17$ (s, 1H, ArH), 7.15 (s, $1 \mathrm{H}, \mathrm{OCHN}), 7.02 \sim 7.05(\mathrm{~m}, 2 \mathrm{H}, \mathrm{ArH}), 6.91(\mathrm{~s}, 1 \mathrm{H}, 3-\mathrm{H})$, $6.83 \sim 6.85$ (dd, $J=2.5,6.5 \mathrm{~Hz}, 1 \mathrm{H}, \mathrm{ArH}), 4.24 \sim 4.41(\mathrm{~m}$, $\left.4 \mathrm{H}, \mathrm{CH}_{2} \mathrm{CH}_{2}\right), 2.21\left(\mathrm{~s}, 3 \mathrm{H}, \mathrm{CH}_{3}\right) ;{ }^{13} \mathrm{C}$ NMR $(125 \mathrm{MHz}$, DMSO- $\left.d_{6}\right) \delta: 175.76,162.47,161.92,156.87,156.64$, $152.63,148.28,131.22,131.07,131.02,129.79,129.35$, $128.59,127.03,125.77,125.69,123.38,123.28,120.28$, $117.02,114.19,112.57,106.49,101.36,91.34,66.91$, 66.80, 40.02, 39.94, 39.85, 39.77, 39.68, 20.73; IR (KBr) $v: 3066,2929,1670,1631,1500,1445,1374,1340,1246$, 1169, 1067, 973, 850, 767, $687 \mathrm{~cm}^{-1}$; ESI-MS m/z: 338.43 (100), 416.59 (45), $592.46\left([\mathrm{M}+\mathrm{H}]^{+}, 52\right)$; HRMS calcd for $\mathrm{C}_{33} \mathrm{H}_{26} \mathrm{~N}_{3} \mathrm{O}_{8} \quad[\mathrm{M}+\mathrm{H}]^{+}$592.1714, found 592.1720.

\section{2 .5 体外抗菌活性测试}

采用打孔测试培养法 ${ }^{[14]}$ : 用灭过菌的打孔器在涂 布菌液的平具上十字对称打 4 个孔, 用无菌微量注射器 分别加入 $100 \mu \mathrm{L}$ 质量浓度为 $500 \mu \mathrm{g} / \mathrm{mL}$ 的样品二甲基 亚砜溶液, 并以 $0.85 \%$ 的无菌生理盐水作对照, 每种菌 做 3 个皿. 将培养血置于恒温培养箱中培养, 其中细菌 平板倒置于 $37{ }^{\circ} \mathrm{C}$ 恒温培养箱中培养 $24 \mathrm{~h}$, 霉菌倒置 于 $28{ }^{\circ} \mathrm{C}$ 恒温培养箱中培养 $48 \mathrm{~h}$, 取出观察有无抑菌作 用. 以校正后的空白对照和处理的菌落平均直径计算抑 制率，计算公式如下：抑制率 $\%=$ (对照药菌落直径一处 理药菌落直径) / 对照药菌落直径 $\times 100 \%$, 其中抑制率 $<$ $30 \%$ 用 “十” 表示, $30 \%<$ 抑制率 $<60 \%$ 用 “+十”, 60\% $<$ 抑制率用 “ +++ ”表示.

辅助材料(supporting Information) 目标化合物的 IR、 ${ }^{1} \mathrm{H} N M R 、{ }^{13} \mathrm{C}$ NMR、ESI-MS 和 HRMS 谱图. 这些材料 可以免费从本刊网站(http://sioc-journal.cn/)上下载. 


\section{References}

[1] Vicente-Sanchez, C.; Egido, J.; Sanchez-Gonzalez, P. D. Food Chem. Toxicol. 2008, 46, 2279.

[2] Yan, X.; Liu, H.-Q.; Zou, Y.-Q.; Ren, Z.-H. Chin. J. Org. Chem. 2008, 28, 1534 (in Chinese)

(延胥, 刘会青, 邹永青, 任占华, 有机化学, 2008, 28, 1534.)

[3] Newell, D.-R.; Searle, K.-M.; Westwood, N.-B.; Burtles, S.-S. Br. J. Cancer 2003, 89, 437

[4] Yan, X.; Xia, L.-L.; Yu, J.; Ding, W.-J. Acta Chim. Sinica 2008, 66, 39 (in Chinese).

(延尔，夏玲玲，于静，丁万见，化学学报, 2008, 66, 39.)

[5] Chen, Z.-G; Wang, C.-N.; Zhao, P.-F., Wang, Y.; Zhou, L.-Y. Acta Chim. Sinica 2010, 68, 2347 (in Chinese). (陈战国，王传宁，赵朋飞，王芸，周利燕，化学学报， 2010， 68, 2347.)

[6] Cui, Z.-N.; Li, Y.; Ling, Y.; Huang, J.; Gui, J.-R.; Wang, R.-Q.; Yang, X.-L. Eur. J. Med. Chem. 2010, 45, 5576.
[7] Jin, Y. X.; Tan, Z. W.; He, M. Z.; Tian, B. H.; Tang, S. X.; Hewlett, I.; Yang, M. Bioorg. Med. Chem. 2010, 18, 2135.

[8] Sevim, R.; Nehir, G.; Habib, E. Farmaco 2002, 57, 171.

[9] Abdel, K. M.; Mohga, M. E.; Nasser, S. A. Molecules 2003, 8, 744.

[10] Wyrzykiewicz, E.; Prukaia, D. J. Heterocycl. Chem. 1998, 35, 381.

[11] Cui, Z.-N.; Zhang, L.; Huang, J.; Ling, Y.; Yang, X.-L. Chin. J. Org. Chem. 2010, 30, 1482 (in Chinese) (崔紫宁, 张莉, 黄娟, 凌云, 杨新玲, 有机化学, 2010, 30, 1482.)

[12] Tang, L.-J. J. Bohai Univ. (Nat. Sci. Ed.) 2007, 28, 310 (in Chinese). (汤立军, 渤海大学学报(自然科学版), 2007, 28, 310.)

[13] Duan, Z.-F; Fu, L.; Li, C.-B. Chem. J. Chin. Univ. 2015, 36, 109 (in Chinese). (段志芳, 付莉, 李充璧, 高等学校化学学报, 2015, 36, 109.)

[14] Qu, L.-B.; Tian, M.-C.; Cheng, S.-X.; Chen, R.-F. Acta Pharm. Sinica 2003, 38, 264 (in Chinese).

(屈凌波, 田孟超, 程森祥, 陈荣峰, 药学学报, 2003, 38, 264.) 